Advances in Radio Science (2003) 1: 93-97

(C) Copernicus $\mathrm{GmbH} 2003$

\title{
Stability and conservation properties of transient field simulations using FIT
}

\section{R. Schuhmann and T. Weiland}

Technische Universität Darmstadt, Dept. of Electrical Engineering and Information Technology, Computational Electromagnetics Laboratory (TEMF), Schloßgartenstr. 8, 64289 Darmstadt, Germany

\begin{abstract}
Time domain simulations for high-frequency applications are widely dominated by the leapfrog timeintegration scheme. Especially in combination with the spatial discretization approach of the Finite Integration Technique (FIT) it leads to a highly efficient explicit simulation method, which in the special case of Cartesian grids can be regarded to be computationally equivalent to the Finite Difference Time Domain (FDTD) algorithm. For stability reasons, however, the leapfrog method is restricted to a maximum stable time step by the well-known Courantcriterion, and can not be applied to most low-frequency applications. Recently, some alternative, unconditionally stable techniques have been proposed to overcome this limitation, including the Alternating Direction Implicit (ADI)-method. We analyze such schemes using a transient modal decomposition of the electric fields. It is shown that stability alone is not sufficient to guarantee correct results, but additionally important conservation properties have to be met.
\end{abstract}

Das Leapfrog-Verfahren ist ein weit verbreitetes Zeitintegrationsverfahren für transiente hochfrequente elektrodynamischer Felder. Kombiniert mit dem räumlichen Diskretisierungsansatz der Methode der Finiten Integration (FIT) führt es zu einer sehr effizienten, expliziten Simulationsmethode, die im speziellen Fall kartesischer Rechengitter als äquivalent zur Finite Difference Time Domain (FDTD) Methode anzusehen ist. Aus Stabilitätsgründen ist dabei die Zeitschrittweite durch das bekannte Courant-Kriterium begrenzt, so dass das LeapfrogVerfahren für niederfrequente Probleme nicht sinnvoll angewendet werden kann. In den letzten Jahren wurden alternativ einige andere explizite oder "halb-implizite" Zeitbereichsverfahren vorgeschlagen, u.a. das "Alternating Direction Implicit" (ADI)-Verfahren, die keiner Beschränkung des Zeitschritts aus Stabilitätsgründen unterliegen. Es zeigt

Correspondence to: R. Schuhmann, T. Weiland

(schuhmann/weiland@temf.tu-darmstadt.de) sich aber, dass auch diese Methoden im niederfrequenten Fall nicht zu sinnvollen Simulationsergebnissen führen. Wie anhand einer transienten Modalanalyse der elektrischen Felder in einem einfachen 2D-Beispiel deutlich wird, ist die Ursache dafür die Verletzung wichtiger physikalischer Erhaltungseigenschaften durch ADI und verwandte Methoden.

\section{Introduction}

Especially in high-frequency field simulations, where one often deals with lossless or at least low-loss structures and a large number of time steps, stability is one of the most important properties of time domain methods, and a required condition for their overall convergence. Here, very often Finite Difference methods (FDTD, Yee (1966)) and the time domain variant of the Finite Integration Technique (FIT, Weiland (1996)) are used, and therein the so-called leapfrog (LF) time stepping algorithm. Based on central difference approximations for the time derivatives in Maxwell's equations, it is known to be conditionally stable - ruled by a maximum stable Courant time step width $\Delta t_{0}-$ and to conserve the electromagnetic energy in lossless structures, if properly defined (Schuhmann and Weiland (2001)).

Since the time step limitation in the LF scheme is coupled to the resolution of the spatial grid, it sometimes leads to strongly oversampled time signals and thus to a poor efficiency of the overall method. As a remedy, some alternative, 'quasi-implicit' approaches have been proposed, such as the Alternating Direction Implicit (ADI) variant of FDTD (Namiki (2000)), or the family of unconditionally stable algorithms proposed in Kole et al. (2001). However, it has been shown by various authors (Darms (2001); Staker and Piket-May (2001); Garcia et al. (2002)) that such schemes may have severe accuracy problems if the Courant limit of the related LF approach is considerably exceeded. In this paper this dissatisfying result is analyzed using an eigenmode decomposition of the transient fields. 


\section{Algebraic Formulation}

\subsection{Basic Equations}

We use here the notation of the FIT (Weiland $(1977,1996)$, where Maxwell's equations are transformed into a set of algebraic equations (linear case, without currents):

$\mu^{-1} \operatorname{curl} \boldsymbol{E}=-\frac{\partial}{\partial t} \boldsymbol{H} \leftrightarrow \quad \mathbf{M}_{\mu}^{-1} \mathbf{C} \widehat{\mathbf{e}}=-\frac{d}{d t} \widehat{\mathbf{h}}$

$\epsilon^{-1} \operatorname{curl} \boldsymbol{H}=\frac{\partial}{\partial t} \boldsymbol{E} \quad \leftrightarrow \quad \mathbf{M}_{\varepsilon}^{-1} \mathbf{C}^{T} \widehat{\mathbf{h}}=\frac{d}{d t} \widehat{\mathbf{e}}$

$\operatorname{div}(\mu \boldsymbol{H})=0 \quad \leftrightarrow \quad \mathbf{S M}_{\mu} \widehat{\mathbf{h}}=\mathbf{0}$

$\operatorname{div}(\epsilon \boldsymbol{E})=\rho \quad \leftrightarrow \quad \widetilde{\mathbf{S}} \mathbf{M}_{\varepsilon} \widehat{\mathbf{e}}=\mathbf{q}$.

The sparse matrices $\mathbf{C}$ and $\mathbf{S}$ are the topological 'curl'-, and 'source'-operators, respectively, and the vectors $\widehat{\mathbf{e}}$ and $\mathbf{h}$ contain the electric and magnetic voltage-type degrees of freedom on a pair of staggered grids. The material matrices $\mathbf{M}_{\varepsilon}^{-1}$ and $\mathbf{M}_{\mu}^{-1}$ are diagonal and positive definite in the simplest case.

An important property of these equations - which can also be used to derive the FDTD-method - is the exact source-free relation of curl-fields,

$\mathbf{S C}=\mathbf{0} \quad$ (primary grid),

$\widetilde{\mathbf{S}} \mathbf{C}^{T}=\mathbf{0} \quad$ (dual grid),

sometimes referred to as consistency properties of the FITdiscretization.

Finally, Eqs. (1) and (2) can be combined to a large system of differential equations for a composite vector $\mathbf{x}$ :

$\frac{d}{d t} \mathbf{x}=\mathbf{A} \mathbf{x}$

with

$\mathbf{x}=\left(\begin{array}{l}\widehat{\mathbf{h}} \\ \widehat{\mathbf{e}}\end{array}\right)$

and

$\mathbf{A}=\left(\begin{array}{cc}\mathbf{0} & -\mathbf{M}_{\mu}^{-1} \mathbf{C} \\ \mathbf{M}_{\varepsilon}^{-1} \widetilde{\mathbf{C}} & \mathbf{0}\end{array}\right)$

The system matrix A can be transformed into a skewsymmetric form using the scaled vectors

$\mathbf{x}^{\prime}=\left(\begin{array}{c}\widehat{\mathbf{h}}^{\prime} \\ \widehat{\mathbf{e}}^{\prime}\end{array}\right) \quad$ with $\quad \widehat{\mathbf{h}}^{\prime}=\mathbf{M}_{\mu}^{1 / 2} \widehat{\mathbf{h}}, \quad \widehat{\mathbf{e}}^{\prime}=\mathbf{M}_{\varepsilon}^{1 / 2} \widehat{\mathbf{e}}$.

Thus, all eigenvalues of $\mathbf{A}$ are purely imaginary, $\lambda_{A, i}=i \omega_{i}$, and all eigenvectors of $\mathbf{A}$ are orthogonal to each other (or can be orthogonalized) referring to

$\left\langle\mathbf{x}_{i}, \mathbf{x}_{j}\right\rangle=\mathbf{x}_{j}^{\prime h} \mathbf{x}_{i}^{\prime}=\widehat{\mathbf{e}}_{j}^{h} \mathbf{M}_{\varepsilon} \widehat{\mathbf{e}}_{i}+\widehat{\mathbf{h}}_{j}^{h} \mathbf{M}_{\mu} \widehat{\mathbf{h}}_{i}=\delta_{i j}$.

\subsection{Time Stepping Schemes}

\subsubsection{Leapfrog (LF) Algorithm}

The leapfrog scheme arises from the allocation of the fields on a staggered time axis and the usage of central difference approximations for the time derivatives:

$\frac{d}{d t} \widehat{\mathbf{h}}^{(n+1 / 2)} \approx \frac{\widehat{\mathbf{h}}^{(n+1)}-\widehat{\mathbf{h}}^{(n)}}{\Delta t}$

$\frac{d}{d t} \widehat{\mathbf{e}}^{(n+1)} \approx \frac{\widehat{\mathbf{e}}^{(n+3 / 2)}-\widehat{\mathbf{e}}^{(n+1 / 2)}}{\Delta t}$

It can be summarized in the update equations

$\mathbf{x}^{(n+1)}=\mathbf{G}_{L F}(\Delta t) \mathbf{x}^{(n)}, \quad \mathbf{x}^{(n)}=\left(\begin{array}{c}\widehat{\mathbf{h}}^{(n)} \\ \widehat{\mathbf{e}}^{(n+1 / 2)}\end{array}\right)$

with the iteration matrix

$\mathbf{G}_{L F}(\Delta t)=\left(\begin{array}{cc}\mathbf{I} & -\Delta t \mathbf{M}_{\mu}^{-1} \mathbf{C} \\ \Delta t \mathbf{M}_{\varepsilon}^{-1} \widetilde{\mathbf{C}} \mathbf{I}-\Delta t^{2} \mathbf{M}_{\varepsilon}^{-1} \widetilde{\mathbf{C}} \mathbf{M}_{\mu}^{-1} \mathbf{C}\end{array}\right)$.

An important property of the LF-operator is the conservation of electric (and magnetic) charges on the discrete level,

$\operatorname{div}\left(\mu \boldsymbol{H}^{(n+1)}\right)=\operatorname{div}\left(\mu \boldsymbol{H}^{(n)}\right)$

$\leftrightarrow \mathbf{S M}_{\mu} \widehat{\mathbf{h}}^{(n+1)}=\mathbf{S M}_{\mu} \widehat{\mathbf{h}}^{(n)}$

$\operatorname{div}\left(\epsilon \boldsymbol{E}^{(n+3 / 2)}\right)=\operatorname{div}\left(\epsilon \boldsymbol{E}^{(n+1 / 2)}\right)$

$\leftrightarrow \widetilde{\mathbf{S}} \mathbf{M}_{\varepsilon} \widehat{\mathbf{e}}^{(n+3 / 2)}=\widetilde{\mathbf{S}} \mathbf{M}_{\varepsilon} \widehat{\mathbf{e}}^{(n+1 / 2)}$

which can be easily proven using Eq. (13) and the matrix properties Eqs. (5) and (6).

\subsubsection{Alternating Direction Implicit (ADI) Algorithm}

The ADI-scheme is based on a splitting of the operator matrix in two parts,

$\mathbf{C}=\mathbf{C}_{1}+\mathbf{C}_{2}$

both of which are used in alternating order in the update equations. This leads to an update scheme in two half-steps, which can be summarized by an iteration matrix

$$
\begin{aligned}
& \mathbf{G}_{A D I}(\Delta t)=\left(\mathbf{I}-\frac{\Delta t}{2} \mathbf{Y}_{1}\right)^{-1}\left(\mathbf{I}+\frac{\Delta t}{2} \mathbf{Y}_{2}\right) \\
& \left(\mathbf{I}-\frac{\Delta t}{2} \mathbf{Y}_{2}\right)^{-1}\left(\mathbf{I}+\frac{\Delta t}{2} \mathbf{Y}_{1}\right)
\end{aligned}
$$

with

$\mathbf{Y}_{1,2}=\left(\begin{array}{cc}\mathbf{I} & -\frac{\Delta t}{2} \mathbf{M}_{\varepsilon}^{-1} \mathbf{C}_{1,2}^{T} \\ \frac{\Delta t}{2} \mathbf{M}_{\mu}^{-1} \mathbf{C}_{1,2} & \mathbf{I}\end{array}\right)$.

For a special choice of the operator splitting Eq. (17), the implicit expressions in Eq. (18) become tridiagonal matrices, which can be efficiently inverted in each time step (see Darms et al. (2003) for more details). 

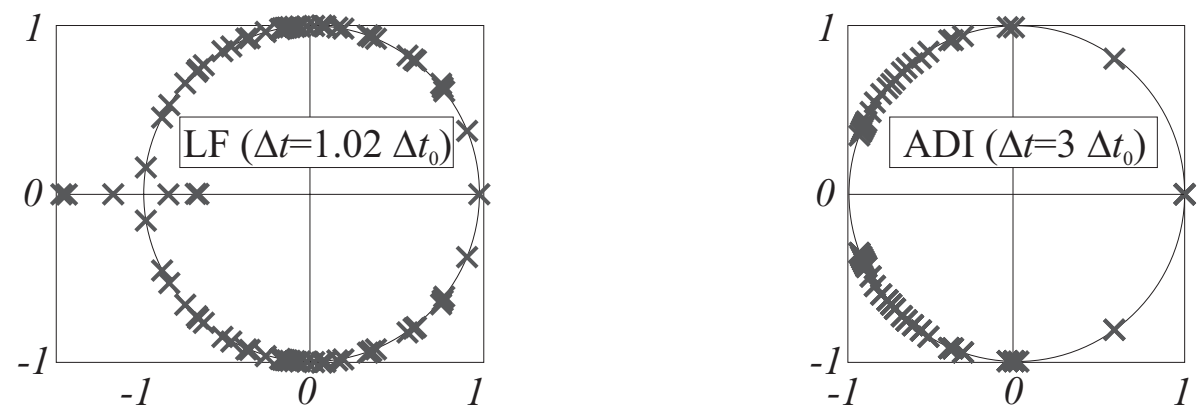

Fig. 1. Complex eigenvalues of the iteration matrices of Leapfrog (LF, left) and ADI (right) for time steps larger than the Courant limit $\Delta t_{0}$. The LF-operator shows some eigenvalues with $\left|\lambda_{G, l f}\right|>1$, causing an instable time integration, whereas all the ADI-eigenvalues lay exactly on the unit cicrle.
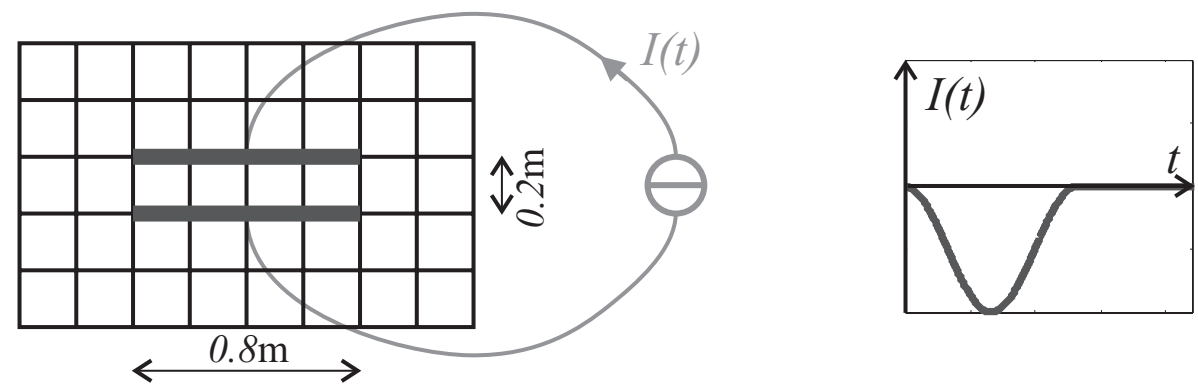

Fig. 2. Model problem: Charging process of a 2D plate capacitor, driven by a $750 \mathrm{kHz}$ current pulse.

\subsubsection{Eigensolutions of the Iteration Matrices}

For the eigenvalues of these iteration matrices one can find the relations

Leapfrog: $\quad\left|\lambda_{G, L F}(\Delta t)\right|=1 \quad \Leftrightarrow \quad \Delta t \leq \Delta t_{0}$

ADI: $\quad\left|\lambda_{G, A D I}(\Delta t)\right|=1 \quad \forall \Delta t>0$,

which are a sufficient condition for the stability of the methods. Whereas the LF method is restricted to time steps below the Courant limit $\Delta t \leq \Delta t_{0}$, the ADI method is unconditionally stable for arbitrary time steps.

In most practical cases the dimension of the iteration matrices is too large to perform a further numerical analysis. For the small test example presented below, however, the matrices are of manageable size, and the results of Eq. (20b) can be visualized as shown in Fig. 1.

For the leapfrog method (left), most of the eigenvalues lay on the unit circle of the complex plane (the stability limit). However, since the time step chosen in this example slightly exceeds the Courant limit $\left(\Delta t=1.02 \Delta t_{0}\right)$, some eigenvalues have left the unit circle at $\lambda_{G}=-1$ and are placed on the negative real axis. The eigenvalues with $\lambda_{G, L F}<-1$ will cause a instable time integration. In the ADI case (Fig. 1, right) all eigenvalues are exactly on the unit circle for arbitrary time steps (here: $\Delta t=3 \Delta t_{0}$ ).

Note that for both methods there is a multiple eigenvalue $\lambda_{G}=1$, referring to so-called static eigenmodes (electro- magnetic fields with eigenfrequency $\omega=0$ ), which will not be changed by the time stepping algorithm.

\section{Transient Modal Expansion}

In the following, the LF and ADI schemes are applied to a 2D (TE) model problem adapted from Garcia et al. (2002). It describes the transient charging process of a simple plate capacitor, driven by a $750 \mathrm{kHz}$ current pulse (cf. Fig. 2).

Figure 3 shows some eigenmodes of the related system matrix A: A static mode (left) with $\nabla \times \boldsymbol{E}=0, \lambda_{A}=0$ and $\lambda_{G}=1$, a second static mode ('plate mode') describing the desired stationary field solution, and a dynamic mode (right) with $\nabla \times \boldsymbol{E} \neq 0, \lambda_{A} \neq 0$, and $\lambda_{G}=e^{i \varphi}$.

During the time stepping process the electric fields can now be decomposed into these (and all the other) eigenmodes. The results of this transient modal expansion, the expansion coefficients as a function of simulation time, are shown in Fig. 4 (Courant time step $\Delta t=\Delta t_{0}$ ) and Fig. 5 (enlarged time step $\Delta t=3 \cdot \Delta t_{0}$ ).

For the Courant time step $\Delta t_{0}$ (Fig. 4) the ADI and LF curves for the plate mode and the dynamic modes are nearly indistinguishable. However, in the ADI simulation arises one more static mode with a magnitude of about $10 \%$ at steady state, which cannot be seen in the LF (reference) results, where all the static modes are below numerical noise. For an enlarged $\Delta t=3 \Delta t_{0}$ (Fig. 5), where no LF results are available any more, the amount of this parasitic field reaches 


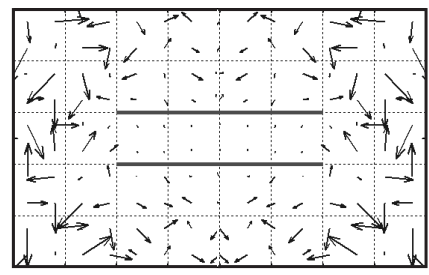

static mode

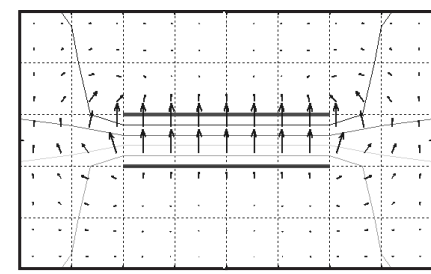

plate mode

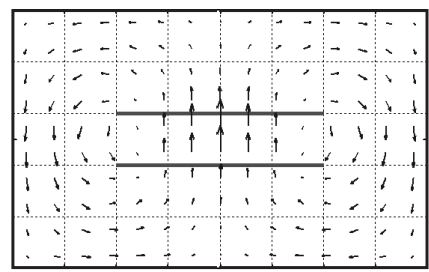

dynamic mode

Fig. 3. Model problem: Electric fields of a static eigenmode (gradient of a random discrete potential vector), the plate mode (stationary solution of charging process), and a dynamic mode (oscillating eigenmode) in the plate capacitor.

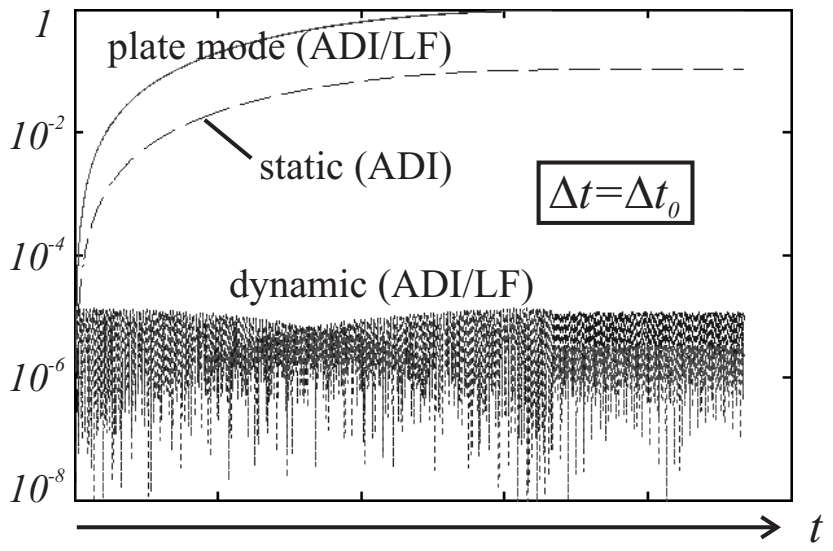

Fig. 4. Transient modal expansion coefficients (logarithmic scale) for the Courant time step $\Delta t=\Delta t_{0}$ : The leapfrog (LF) curves (which serve as a reference result) show the desired plate mode and some dynamic modes excited by the charging process, whereas all static modes are below round off. The ADI curves, however, include a parasitic static mode with a magnitude of about $10 \%$ of the total field at steady state.

the order of magnitude of the desired field solution (plate mode). This unphyiscal mode also qualitatively disturbes the stationary field solution, which is not shown here.

As the reason for this behaviour of ADI we postulate here the loss of orthogonality between the eigenvectors of the iteration matrix: In the leapfrog scheme it can be easily shown that the electric part of the eigenvectors of the iteration matrix $\mathbf{G}_{L F}$ and the system matrix $\mathbf{A}$ are identical, and that they fulfill the same orthogonality condition Eq. (11). In the ADI scheme, however, this property is no longer valid: Although the static modes of $\mathbf{G}_{A D I}$ remain unchanged compared to the static solutions of $\mathbf{A}$ ( or $\mathbf{G}_{L F}$ ) - this can be proven for the 2D TE-case analyzed here - the dynamic modes now include parts of the original static modes. As a consequence, energy can be transferred in each time step between these two classes of eigensolutions (which should be exactly separated in continous theory). As a visualization of this fact, Fig. 6 shows the orthogonality pattern of the ADI-matrix for $\Delta t=3 \cdot \Delta t_{0}$, exhibiting the parasitic coupling between static and dynamic solutions. As another consequence of this loss of consistency in the discrete model, the field solutions

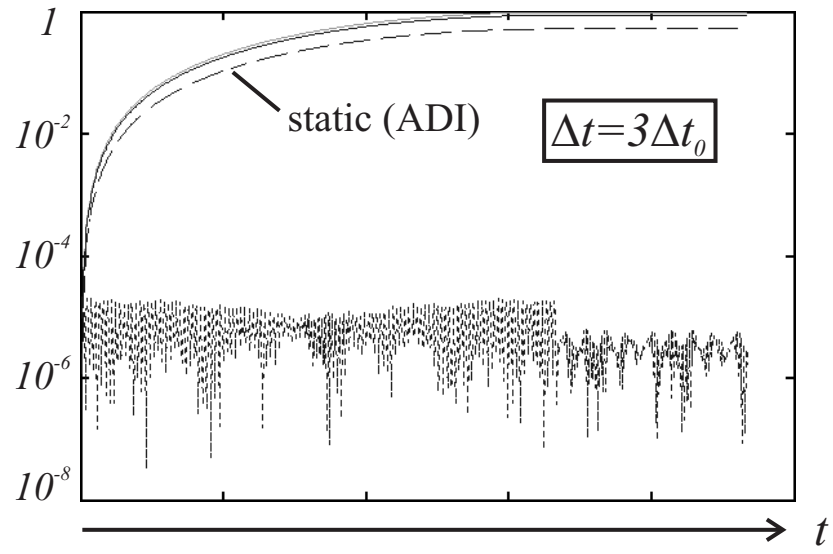

Fig. 5. Transient modal expansion coefficients (logarithmic scale) for enlarged time step $\Delta t=3 \cdot \Delta t_{0}$ : The magnitude of the parasitic static mode in the ADI solution has considerably increased. No Leapfrog solutions are available abouve the Courant limit (instable time integration).

of ADI are no longer source-free, but parasitic electric and magnetic charges arise during the iteration. The intrinsic algebraic reason for these results is the splitting of the operator matrix $\mathbf{C}=\mathbf{C}_{1}+\mathbf{C}_{2}$ in the derivation of the ADI-update equations. Since the spatial curl operator does no longer appear as a whole in the update process, the consistency properties Eqs. (5) and (6) are not applicable any more.

All these effects increase with a growing time step $\Delta t$. Although the ADI-method itself shows a 2nd order convergence for $\Delta t \rightarrow 0$ (cf. also Garcia et al. (2002)), the results in this analysis show that the parasitic effect can be observed also for moderate time steps, even in the range of the Courant limit of the leapfrog method.

\section{Conclusion}

A transient modal expansion of the electric field in time domain methods has been used to analyze different time stepping approaches. Using this powerful tool it can be shown that the ADI method, although being unconditionally stable, 


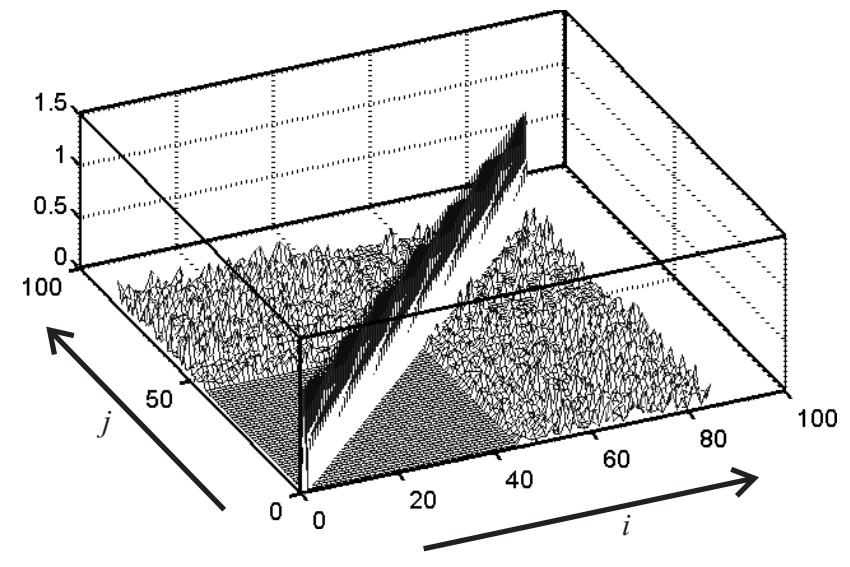

Fig. 6. Orthogonality pattern $\left\langle\mathbf{x}_{i}, \mathbf{x}_{j}\right\rangle \neq \delta_{i j}$ of the eigenmodes $\mathbf{x}_{i}$ of the ADI iteration matrix. The (degenerated) static modes can be orthogonalized, but the orthogonality between static and dynamic modes is lost. This unphysical effect increases with the time step width (here: $\Delta t=3 \cdot \Delta t_{0}$ ).

does not conserve the energy of the original dynamic eigenmodes, even for moderate time steps. The main reason for this behaviour is the operator splitting in the construction of the ADI scheme, which leads to a loss of important consistency properties and of the orthogonality of the system's eigensolutions. The same poor results also have to be expected for similar schemes which are based on the idea of splitting the spatial operators to obtain unconditional stability of the time integration.

\section{References}

Darms, M.: Analyse des ADI Zeitintegrationsverfahrens für zeitlich veränderliche Feldprobleme, Studienarbeit, Technische Universität Darmstadt, 2001.

Darms, M., Schuhmann, R., Spachmann, H., and Weiland, T.: Dispersion and Asymmetry Effects of ADI-FDTD, IEEE Microwave and Wireless Components Letters, in press, 2003.

Garcia, S. G., Lee, T., and Hagness, S. C.: On the Accuracy of the ADI-FDTD Method, IEEE Antennas and Wireless Propagation Letters, 1, 31-34, 2002.

Kole, J. S., Figge M. T., and De Raedt, H.: Unconditionally Stable Algorithms to Solve the Time-Dependent Maxwell Equations, Physical Review E, 64, 066705, 2001.

Namiki, T.: 3-D ADI-FDTD Method - Unconditionally Stable Time-Domain Algorithm for Solving Full Vector Maxwell's Equations, IEEE Trans. on Microwave Theory and Techniques, 48, 1743-1748, 2000.

Schuhmann, R. and Weiland, T.: Conservation of Discrete Energy and Related Laws in the Finite Integration Technique, Progress In Electromagnetics Research, PIER, 32, 301-316, 2001.

Staker, S. and Piket-May, M.: Algorithm Study of ADI-FDTD, Digest of the 2001 USNC/URSI National Radio Science Meeting, Boston, 255, 2001.

Weiland, T.: A Discretization Method for the Solution of Maxwell's Equations for Six-Component Fields, Electronics and Communication (AEÜ), 31, 116, 1977a.

Weiland, T.: Time Domain Electromagnetic Field Computation with Finite Difference Methods, Int. Journal of Numerical Modelling, 9, 295-319, 1996.

Yee, K. S.: Numerical Solution of Initial Boundary Value Problems Involving Maxwell's Equations in Isotropic Media, IEEE Trans. on Antennas and Propagation, 17, 585-589, 1966. 